\title{
Three-in-one: GPS-IR measurements of ground surface elevation changes, soil moisture, and snow depth at a permafrost site in the northeastern Qinghai-Tibet Plateau
}

Jiahua Zhang ${ }^{1}$, Lin Liu ${ }^{1}$, Lei $\mathrm{Su}^{2,3}$, Tao $\mathrm{Ch}^{2,4}$

$5 \quad$ 'Earth System Science Programme, Faculty of Science, The Chinese University of Hong Kong, Hong Kong, 999077, China ${ }^{2}$ Heihe Remote Sensing Experimental Research Station, Key Laboratory of Remote Sensing of Gansu Province, Northwest Institute of Eco-Environment and Resources, Chinese Academy of Sciences, Lanzhou, 730000, China ${ }^{3}$ University of Chinese Academy of Sciences, Beijing, 100049, China 10

${ }^{4}$ Center for Excellence in Tibetan Plateau Earth Sciences, Chinese Academy of Sciences, Beijing, 100101, China

Correspondence to: Tao Che (chetao@1zb.ac.cn)

\section{Supplementary}

We assess the monument stability of QLBG and its impact on the GPS-IR-measured surface elevation changes and soil moisture content during DOY 182-243 in 2018. We noted in section 2 that the foundation depth of QLBG is slightly

15 shallower than the active layer base. The monument might settle when the soil around the foundation thaws in late summer. We can obtain the vertical monument movement from the positioning observations of QLBG and use them to examine the monument stability and the necessity of correcting the GPS-IR measurements.

We compute the QLBG's positioning measurements during DOY 182-243 in 2018 in the local NEU (north, east, and up) coordinate reference system, by using GAMIT/GLOBK (http://geoweb.mit.edu/gg/links.php) and four International GNSS Service reference stations BJFS $\left(39.61^{\circ} \mathrm{N}, 115.89^{\circ} \mathrm{E}\right), \mathrm{JFNG}\left(86.54^{\circ} \mathrm{N}, 114.49^{\circ} \mathrm{E}\right), \mathrm{URUM}\left(43.81^{\circ} \mathrm{N}, 87.60^{\circ} \mathrm{E}\right)$, and $\mathrm{LHAZ}$ $\left(29.66^{\circ} \mathrm{N}, 91.10^{\circ} \mathrm{E}\right)$. We show the positioning results in Fig. S1. 


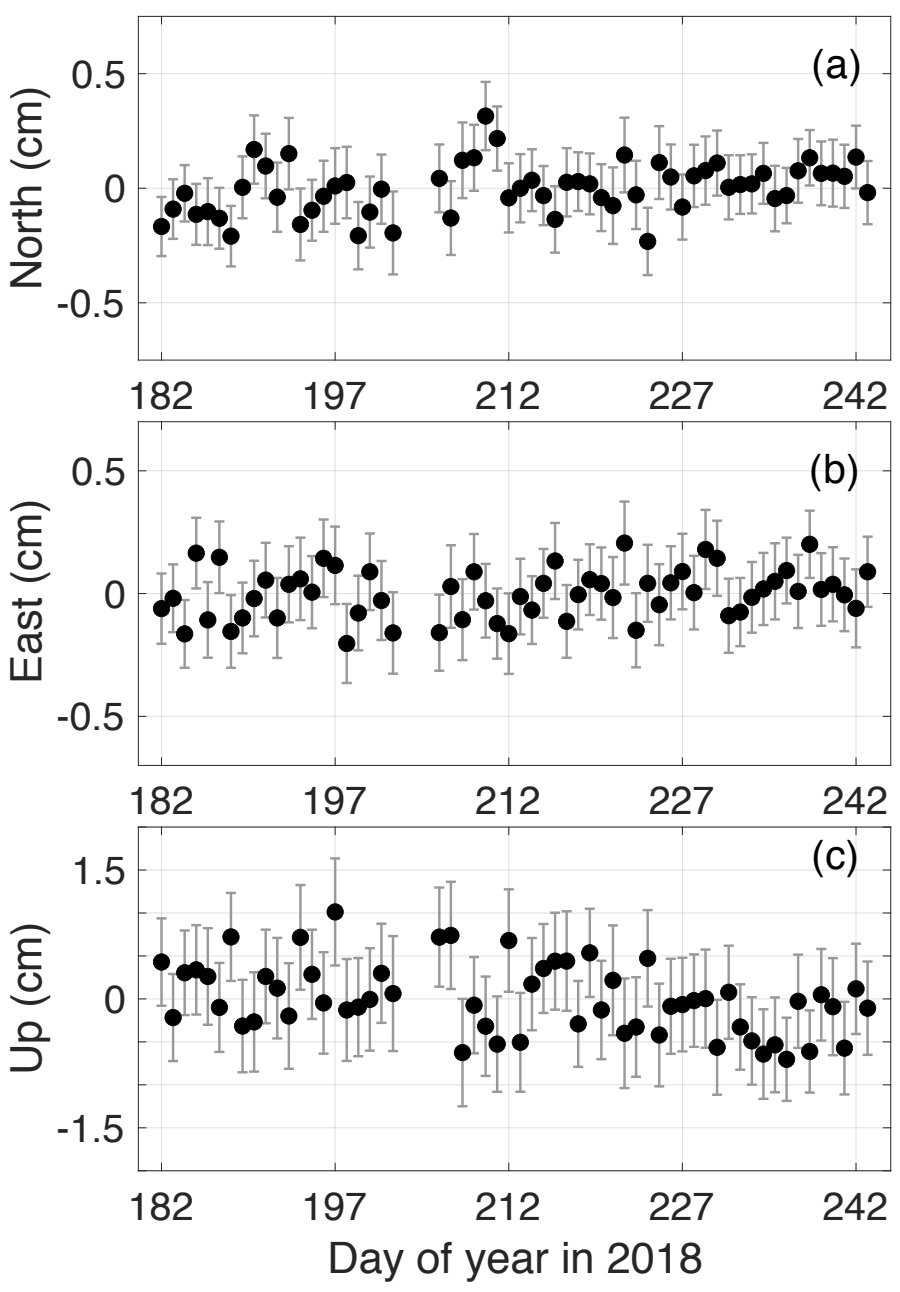

25 Figure S1: Positioning observations of QLBG in the (a) north, (b) east, and (c) up directions during DOY $182-243$ in 2018. They are referenced to their mean values individually.

For the positionings in the east and north directions, their variations are as small as in the range of less than $1 \mathrm{~cm}$. It implies that the monument has no significant inclination and the antenna is still in up-right direction. The positionings in the up

30 direction are composed of the monument displacements and solid earth movement, such as crustal motion and surface deformation due to atmospheric and hydrological surface loadings. Regarding the crustal movement, the velocity field of the contemporary crustal motion of the QTP relative to the Eurasian plate has been constructed (Gan et al., 2005; Liang et al., 2013). The magnitude of the uplift rate of our study site is less than $1 \mathrm{~mm} /$ year. The vertical crustal movement during DOY 
182-243 in 2018 was negligible. We use the simulations of surface deformation provided by ESMGFZ (Earth System

35 Modelling group of GFZ, Dill and Dobslaw, 2013) to examine the influence of the surface loadings. The simulated values are shown in Fig. S2. During DOY 182-243 in 2018, the variation range of the surface deformation due to atmosphere was around $9 \mathrm{~mm}$, and that of continental hydrology was $4 \mathrm{~mm}$. We remove the contributions of the surface loadings from the positionings and use the residuals as the vertical monument movement, which are shown in Fig. S3. The monument movement is relatively noisy, and varies in a range with a magnitude of $1.5 \mathrm{~cm}$. We build a best linear fit for them and find

40 that they do not present a statistically significant trend (The slope of the linear fit line is $0.003 \pm 0.004 \mathrm{~cm} /$ day, which is not statistically significant $(\alpha=0.05)$ ). We also find that the movement follows a Gaussian normal distribution with the mean of $0 \mathrm{~cm}$ and the standard deviation of $0.42 \mathrm{~cm}$. The monument movement barely has an impact on the constant $H_{0}$. And they have little impact on the GPS-IR-measured surface elevation changes, as we focus on the temporal variation of the surface elevation.
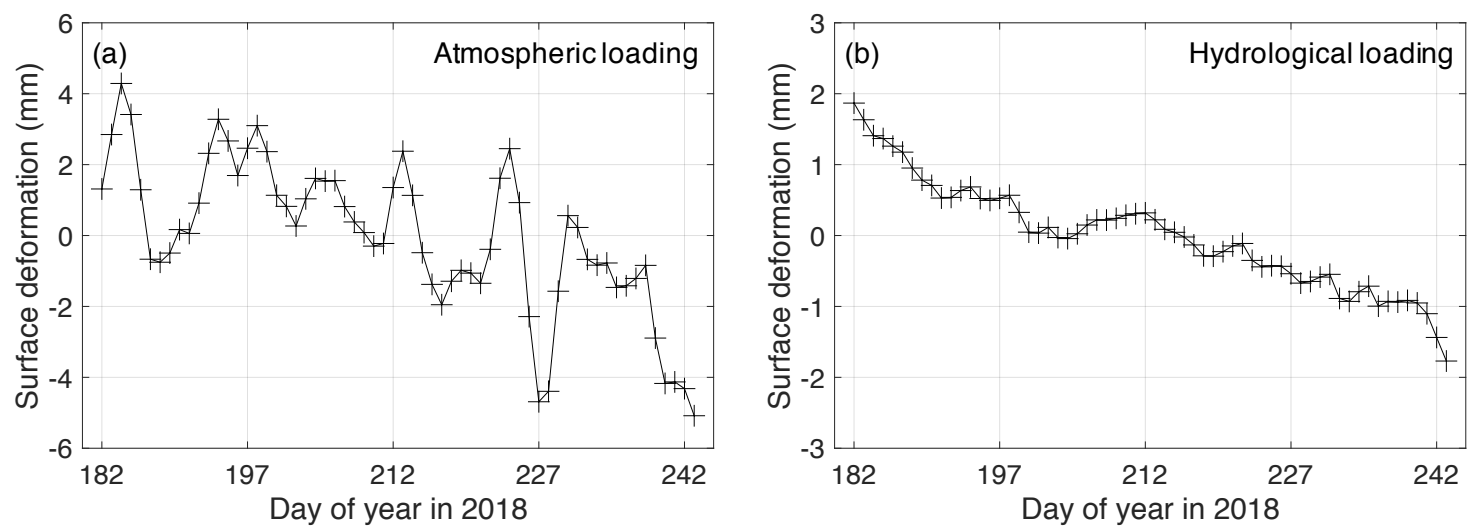

Figure S2: Surface deformation due to (a) atmosphere and (b) continental hydrology, respectively, during DOY 182-243 in 2018. They are referenced to their mean values individually. 

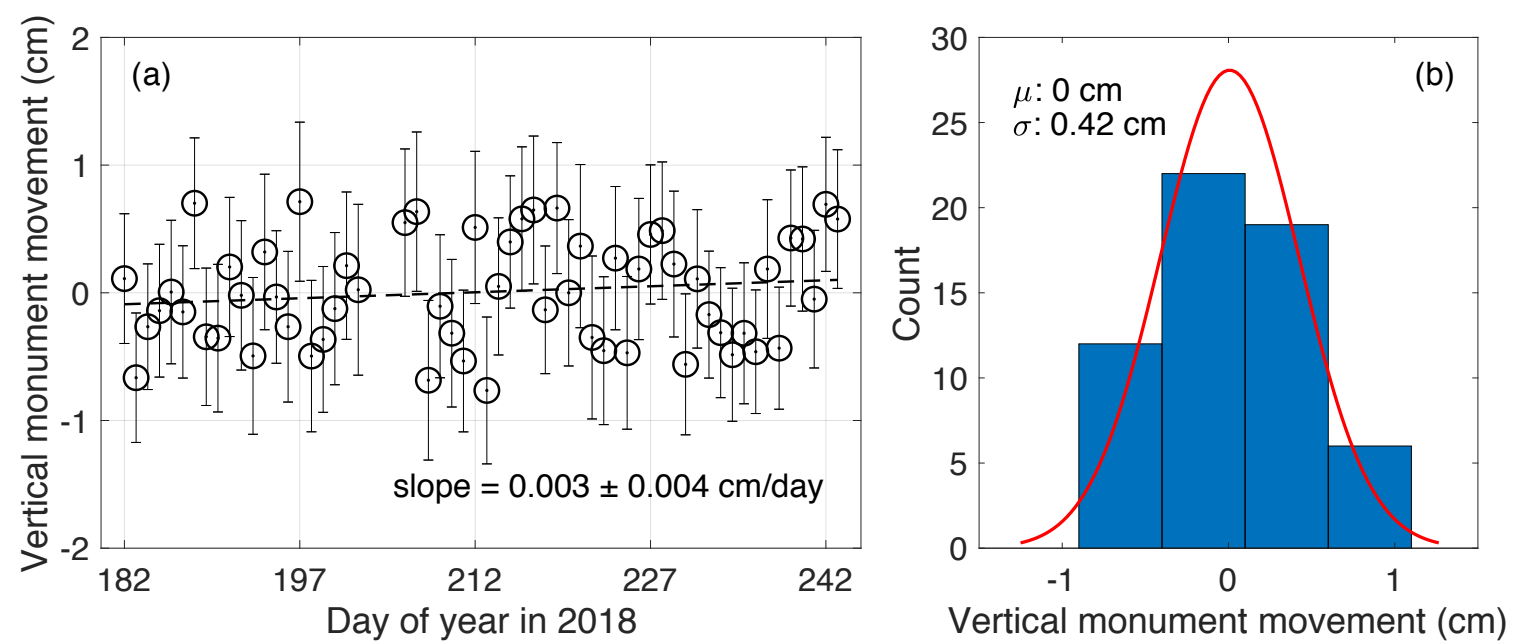

Figure S3: (a) Time series of the vertical monument movement during DOY 182-243 in 2018 . Their best linear fit line is also presented (as a dashed line) with the slope of $0.003 \pm 0.004 \mathrm{~cm} /$ day. (b) Histogram of the vertical monument movement shown in (a) and their normal distribution fit (red curve). The mean $(\mu)$ and standard deviation $(\sigma)$ of this normal fit are $0 \mathrm{~cm}$ and $0.42 \mathrm{~cm}$, respectively.

In the modified method, the time-varying $H_{0}{ }^{\prime}$ is composed of the constant $H_{0}$ and the modeled surface elevation changes. Here, we assess the impact of the vertical monument movement on the modeled surface elevation changes through affecting model parameters. When considering the vertical movement (denoted as $v$ hereafter), based on equation (5), the model

60 expression is changed to

$d(t)+v(t)=d_{s} \widetilde{I_{T}}+d_{0}$,

Studying the impact of $v$ on the model parameters is essentially to investigate the dependence of $v$ on $\widetilde{I_{T}}$. We build a scatter plot between $v$ and $\widetilde{I_{T}}$, which is shown in Fig. S4. The correlation coefficient between them is as weak as 0.14 . This result can be expected as the vertical movement is almost white noise whereas the thermal indices possess an increasing trend. The

65 weak correlation indicates that the monument movement has little impact on the estimates of $d_{s}$ and $d_{0}$, then the modeled surface elevation changes. Together with the discussion in the last paragraph, the vertical monument movement has little impact on the time-varying reflector height $H_{0}{ }^{\prime}$, thus barely affect the phases and soil moisture estimates by our method. 


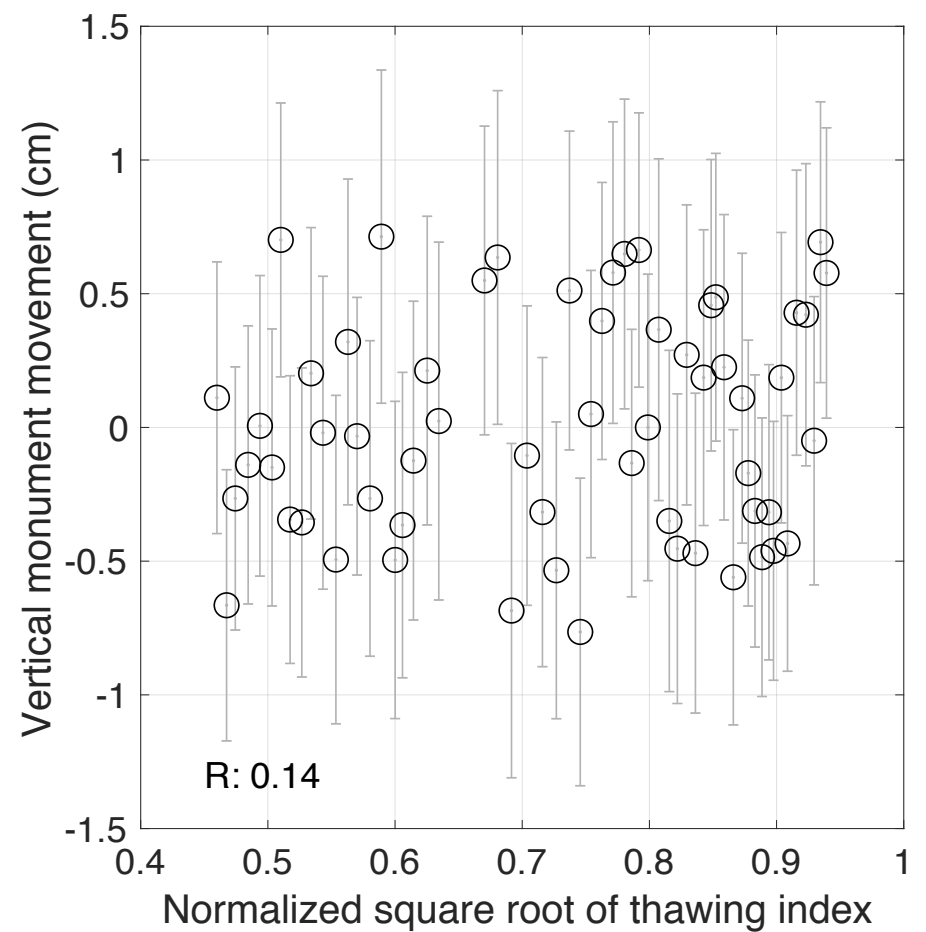

Figure S4: Scatter plot between square root of normalized thawing index and the vertical monument movement $v$ during DOY 182-243 in 2018. 